\title{
SPHERE THEOREM BY MEANS OF THE RATIO OF MEAN CURVATURE FUNCTIONS
}

\author{
SUNG-EUN KOH \\ Department of Mathematics, Konkuk University, Seoul, 143-701, Korea \\ e-mail:sekoh@kkucc.konkuk.ac.kr
}

(Received 2 June, 1998)

\begin{abstract}
It is well known that a compact embedded hypersurface of the Euclidean space without boundary is a round sphere if one of mean curvature functions is constant. In this note, we show that a compact embedded hypersurface of the Euclidean space (and other constant curvature spaces) without boundary is a round sphere if the ratio of some two mean curvature functions is constant.
\end{abstract}

1991 Mathematics Subject Classification. 53C40, 53C42

1. Introduction. Let $M^{n}$ be an emdedded submanifold of $N^{n+1}$ and let $H_{k}$ denote the $k$-th mean curvature function of $M^{n}$, that is, $H_{k}$ is the $k$-th elementary symmetric polynomial of principal curvatures of $M^{n}$ divided by $\left(\begin{array}{l}n \\ k\end{array}\right)$, and $H_{0}$ is defined to be 1 . For instance, $H_{1}$ is the usual mean curvature and $H_{n}$ is the GaussKronecker curvature.

Alexandrov's well-known sphere theorem [1] states that, when $N^{n+1}$ is the Euclidean space $\mathbb{R}^{n+1}$, the hyperbolic space $\mathbb{M}^{n+1}$ or the open half sphere $\mathbb{S}_{+}^{n+1}, M^{n}$ is a round sphere if $H_{1}$ is constant. This theorem was generalized in [3] in the following way.

Theorem A. Let $N^{n+1}$ be one of $\mathbb{R}^{n+1}$, $\mathbb{H}^{n+1}$ or $\mathbb{S}_{+}^{n+1}$ and let $\phi: M^{n} \rightarrow N^{n+1}$ be an isometric embedding of a compact oriented n-dimensional manifold without boundary $M^{n}$. If $H_{k}$ is constant for some $k=1,2, \ldots, n$, then $\phi\left(M^{n}\right)$ is a geodesic hypersphere.

In this note, we generalize Theorem $\mathrm{A}$ in the following way.

Theorem B. Let $N^{n+1}$ be one of $\mathbb{R}^{n+1}$, $\mathbb{H}^{n+1}$ or $\mathbb{S}_{+}^{n+1}$ and $\phi: M^{n} \rightarrow N^{n+1}$ be an isometric embedding of a compact oriented n-dimensional manifold without boundary $M^{n}$. If the ratio $H_{k} / H_{l}$ is constant for some $k, l=0,1,2 \ldots, n, k>l$ and $H_{l}$ does not vanish on $M^{n}$, then $\phi\left(M^{n}\right)$ is a geodesic hypersphere.

As $H_{0}$ is defined to be 1 , the above theorem reduces to Theorem $\mathrm{A}$ if $l=0$. Theorem B is a generalization of Theorem A in this sense. Note also that we cannot expect the result for the whole sphere $\mathbb{S}^{n+1}$. For example, $H_{1}$ and $H_{2}$ of the embedding

$$
\mathbb{S}^{1}(a) \times \mathbb{S}^{1}(b) \subset \mathbb{S}^{3}, \quad a^{2}+b^{2}=1, \quad a \neq b,
$$

are nonzero constants. 
2. Proof. We use the hyperboloid model for $\mathbb{H}^{n+1}$ and the usual embedding of $\mathbb{S}^{n+1}$ into $\mathbb{R}^{n+2}$. Let $\eta$ denote a unit normal field on $M^{n}$. We use the following Minkowski formula (for proof, see [3]) where $\langle$,$\rangle denotes the usual Euclidean inner$ product on $\mathbb{R}^{n+1}$ (on $\mathbb{R}^{n+2}$ ) when $N^{n+1}$ is $\mathbb{R}^{n+1}$ (when $N^{n+1}$ is $\mathbb{S}_{+}^{n+1}$ ) and the Lorentzian inner product on $\mathbb{R}^{n+2}$ when $N^{n+1}$ is $\mathbb{H}^{n+1}$.

Lemma A. The following identities hold for every $k=1, \ldots, n$.

(i) When $N^{n+1}$ is $\mathbb{R}^{n+1}$,

$$
\int_{M}\left(H_{k-1}+H_{k}\langle\phi, \eta\rangle\right) d M=0 .
$$

(ii) When $N^{n+1}$ is $\mathfrak{H}^{n+1}$,

$$
\int_{M}\left(H_{k-1}\langle\phi, p\rangle+H_{k}\langle\eta, p\rangle\right) d M=0 \text { for any } p \in \mathbb{R}^{n+2} \text {. }
$$

(iii) When $N^{n+1}$ is $\mathbb{S}_{+}^{n+1}$,

$$
\int_{M}\left(H_{k-1}\langle\phi, p\rangle-H_{k}\langle\eta, p\rangle\right) d M=0 \text { for any } p \in \mathbb{R}^{n+2} \text {. }
$$

We also use the following inequalities for higher order mean curvatures.

Lemma B. Suppose $H_{k}>0$ for some $k=1,2, \ldots, n$. Then the following hold.

(i) $H_{k}^{\frac{k-1}{k}} \leq H_{k-1}$; hence every $H_{l}, l \leq k$, is positive.

(ii) $H_{k} / H_{k-1} \leq H_{k-1} / H_{k-2}$.

(iii) For every $l<k, \quad H_{k} / H_{l} \leq H_{k-1} / H_{l-1}$.

Proof of Lemma B. For (i), (ii), see, for example, [2, Section 12].

From (ii), we have

$$
H_{k} / H_{k-1} \leq H_{k-1} / H_{k-2} \leq \cdots \leq H_{l+1} / H_{l} \leq H_{l} / H_{l-1},
$$

which is equivalent to (iii).

Now, assume

$$
H_{k} / H_{l}=\alpha
$$

for a constant number $\alpha$.

(2.1). Proof when $N^{n+1}=\mathbb{R}^{n+1}$. Since $M^{n}$ is compact, one can find a point in $M^{n}$ where all the principal curvatures are positive. Then $H_{k}, H_{l}$ are positive at that point. Since $H_{k} / H_{l}$ is constant on $M^{n}$ and since $H_{l}$ does not vanish on $M^{n}$ by 
assumption, $H_{k}$ and $H_{l}$ are positive on $M^{n}$. Then $\alpha>0$ and from the inequality (ii) of Lemma B, we have

$$
0<\alpha=H_{k} / H_{l} \leq H_{k-1} / H_{l-1} .
$$

Since $H_{k}=\alpha H_{l}$, we have by Lemma A,

$$
\begin{aligned}
0 & =\int_{M}\left(H_{k-1}+H_{k}\langle\phi, \eta\rangle\right) d M \\
& =\int_{M}\left(H_{k-1}+\alpha H_{l}\langle\phi, \eta\rangle\right) d M,
\end{aligned}
$$

that is,

$$
\int_{M} H_{k-1} d M=\int_{M}\left(-\alpha H_{l}\langle\phi, \eta\rangle\right) d M
$$

On the other hand, since $\alpha$ is constant, we also have by Lemma A,

$$
\int_{M} \alpha\left(H_{l-1}+H_{l}\langle\phi, \eta\rangle\right) d M=0,
$$

that is,

$$
\int_{M} \alpha H_{l-1} d M=\int_{M}\left(-\alpha H_{l}\langle\phi, \eta\rangle\right) d M .
$$

From (1) and (2), we have

$$
\int_{M}\left(H_{k-1}-\alpha H_{l-1}\right) d M=0 .
$$

Since we have from $(*)$

$$
H_{k-1}-\alpha H_{l-1} \geq 0
$$

it follows that

$$
H_{k-1} / H_{l-1}=\alpha=H_{k} / H_{l}
$$

everywhere on $M^{n}$. Thus, proceeding inductively, we have finally

$$
H_{k-l}=H_{k-l} / H_{0}=\alpha
$$

everywhere on $M^{n}$. Thus, by Theorem A, $\phi\left(M^{n}\right)$ is a geodesic hypersphere.

(2.2) Proof when $N^{n+1}=\mathbb{H}^{n+1}$ : At a point of $M^{n}$ where the distance function of $\mathbb{H}^{n+1}$ attains its maximum, all the principal curvatures are positive. Then $H_{k}, H_{l}$ are positive on $M^{n}$ and (*) also holds in this case. Since $H_{k}=\alpha H_{l}$, we have 


$$
\begin{aligned}
0 & =\int_{M}\left(H_{k-1}\langle\phi, p\rangle+H_{k}\langle\eta, p\rangle\right) d M \\
& =\int_{M}\left(H_{k-1}\langle\phi, p\rangle+\alpha H_{l}\langle\eta, p\rangle\right) d M,
\end{aligned}
$$

that is,

$$
\int_{M} H_{k-1}\langle\phi, p\rangle d M=\int_{M}\left(-\alpha H_{l}\langle\eta, p\rangle\right) d M
$$

Since $\alpha$ is constant, we also have

$$
\int_{M} \alpha\left(H_{l-1}\langle\phi, p\rangle+H_{l}\langle\eta, p\rangle\right) d M=0
$$

so that

$$
\int_{M}\left(H_{k-1}-\alpha H_{l-1}\right)\langle\phi, p\rangle d M=0
$$

Now, if we take $p=(1,0, \ldots, 0) \in \mathbb{R}^{n+2}$, then the sign of $\langle\phi, p\rangle$ does not change on $M^{n}$. Since $H_{k-1}-\alpha H_{l-1} \geq 0$ from $(*)$, we have

$$
H_{k-1} / H_{l-1}=\alpha=H_{k} / H_{l}
$$

everywhere on $M^{n}$. Thus, proceeding inductively, we have finally

$$
H_{k-l}=H_{k-l} / H_{0}=\alpha
$$

everywhere on $M^{n}$. Thus, by Theorem $\mathrm{A}, \phi\left(M^{n}\right)$ is a geodesic hypersphere.

(2.3) Proof when $N^{n+1}=\mathbb{S}_{+}^{n+1}$. Let $c \in \mathbb{S}^{n+1}$ be the centre of $\mathbb{S}_{+}^{n+1}$. Then at a point of $M^{n}$ where the height function $\langle\phi, c\rangle$ attains its maximum, all the principal curvatures are positive because $M^{n}$ lies in the open half sphere with the centre $c$. Now proceeding as in (2.2), we have

$$
\int_{M}\left(H_{k-1}-\alpha H_{l-1}\right)\langle\phi, p\rangle d M=0 .
$$

Since $M^{n}$ lies in the open half sphere, one can find a vector $p \in \mathbb{R}^{n+2}$ so that $\langle\phi, p\rangle$ is positive on $M^{n}$. Since $H_{k-1}-\alpha H_{l-1} \geq 0$ by $(*)$, arguing in the same way as before, we can see that $H_{k-l}=\alpha$, that is, $\phi\left(M^{n}\right)$ is a geodesic hypersphere.

AcKnOwLEDGEMEnTs. This research was supported by the KOSEF 96-0701-0201-3, by BSRI-97-1438 and by Konkuk University in 1998. The author would like to thank Professor Hong-Jong Kim for his interest in this paper. 


\section{REFERENCES}

1. A. D. Alexandrov, A characteristic property of spheres, Ann. Mat. Pura Appl. 58 (1962), 303-315.

2. E. F. Beckenbach and R. Bellman, Inequalities (Springer-Verlag, Berlin, 1971).

3. S. Montiel and A. Ros, Compact hypersurfaces: the Alexandrov theorem for higher order mean curvatures, in Differential geometry (B. Lawson, ed.), Pitman Monographs 52 (Longman, New York, 1991), 279-296. 